\title{
PROGRESS REPORT ON THE SMALL ISOCHRONOUS RING PROJECT AT NSCL *
}

\author{
E. Pozdeyev, R. Fontus, D. Lawton, F. Marti, J. Rodriguez, \\ D. Sanderson, R.C. York, A. Zeller, R. Zink \\ NSCL, MSU, East Lansing, MI 48824-1341, USA
}

\begin{abstract}
The small Isochronous Ring (SIR), whose main objectives are experimental studies of space charge effects in the isochronous regime and validation of space charge codes, is under development at the National Superconducting Cyclotron Laboratory (NSCL) at Michigan State University (MSU). The ring is a small-scale experiment that simulates the dynamics of intense beams in large-scale accelerators. It will store hydrogen and deuterium ions at energies of approximately $20-30 \mathrm{keV}$ for a few tens of turns. The low beam energy and the small scale of the experiment provide a unique opportunity to perform accurate experiments on space charge dominated beams that are difficult to conduct in large-scale accelerators because of power and timing limitations imposed on beam diagnostics. The paper reports the status of the project and describes the progress in the development of ring subsystems.
\end{abstract}

\section{INTRODUCTION}

In recent years, there has been a increased interest in space charge effects in isochronous cyclotrons. Inspired by successful high-current operation of the Ring Cyclotron at PSI, several authors proposed to use an isochronous cyclotron as a driver for a number of applications [1],[2],[3] including radioactive waste transmutation, energy production with accelerator driven nuclear reactors, generation of neutrons and other secondary particles, etc. Tentative designs of such a machine have a maximum beam energy of $1 \mathrm{GeV}$ and a beam current of $10 \mathrm{~mA}$, yielding a total beam power of $10 \mathrm{MW}$.

Successful operation of a $10 \mathrm{MW}$ cyclotron would be impossible without a deep understanding of space charge effects in the isochronous regime. There is a tremendous demand for experimental data that can be extrapolated to predict the evolution of beams of higher intensity. This experimental data can be also used to validate codes that are used for simulations of space charge effects in cyclotrons and other types of machines.

Detailed measurement of beam parameters in existing high current machines is difficult because of power and timing requirements on diagnostics. We developed the Small Isochronous Ring (SIR) project ([4],[5],[6]) at Michigan State University to study space charge effects in circular machines working in or close to the isochronous regime. The ring is a small-scale experiment that requires low beam intensities to simulate the dynamics of intense

\footnotetext{
* Work supported by NSF Grant PHY 0110253
}

beams in large-scale accelerators. The important issues to be addressed by the ring are the space charge induced vortex motion specific to the isochronous regime, the longitudinal break-up of long bunches, formation of the selfconsistent stable charge distribution by short bunches, and formation of weak beam tails and beam halo. The results of the experiments will have applications to high current isochronous cyclotrons and synchrotrons at the transition gamma. The possibility of accurate measurement of beam parameters will also allow SIR to be used as a convenient tool for validation of multi-particle codes used for space charge simulations.

\section{DESIGN FEATURES}

The SIR lattice consists of four $90^{\circ}$ dipole magnets with edge focusing. The edge focusing provides both vertical focusing and isochronism in the ring. Because the ring is isochronous there is no need for an RF system to keep the beam bunched. However, an accelerating gap can later be installed in the ring for experiments with high intensity beams in a non-isochronous regime. In the operational ring, a pulsed electrostatic deflector will be used to send the beam to a fast Faraday Cup after a chosen number of turns. The longitudinal beam profile will be measured by the Faraday Cup with a time resolution of 1 ns that corresponds to a spatial resolution of 1-2 mm. Examination of the beam profile measured after a different number of turns can provide better insight into space charge effects in the isochronous regime.

Figure 1shows a photograph of the project taken in May, 2003. Table 1 lists main SIR parameters. More details on the SIR lattice and beam dynamics in the ring can be found in [7].

\section{Ion source, injection line, and injection system}

A multi-cusp ion source that can be biased up to $30 \mathrm{keV}$ is used for production of hydrogen beams. The source generates three mass states: $\mathrm{H}^{+}, \mathrm{H}_{2}^{+}$, and $H_{3}^{+}$. A dipole magnet situated $70 \mathrm{~cm}$ below the ion source separates the species and sends a selected beam towards the ring. The dipole is identical to the four magnets that form the ring. The injection line also includes an Einzel lens, a beam diagnostics box, electrostatic deflector plates, a quadrupole triplet that matches the injection line with the ring, and a beam chopper.

The beam is injected into the ring by a pulsed electrostatic inflector. Voltage on the inflector plates is regulated 


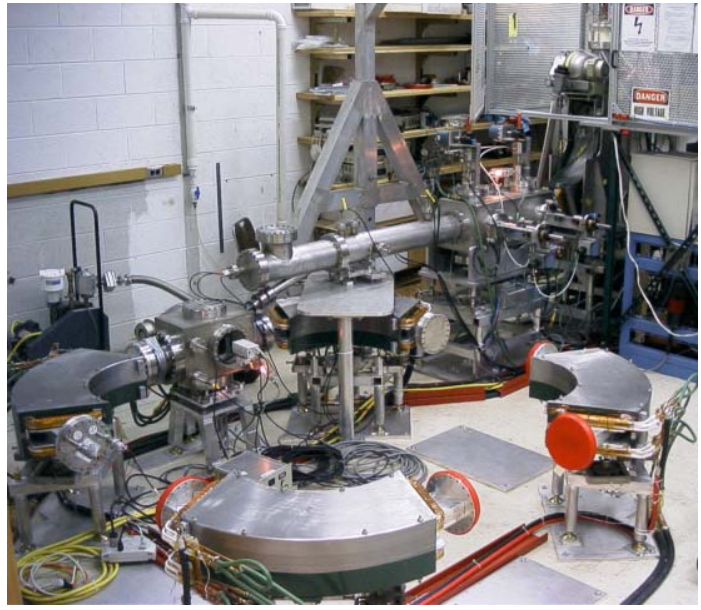

Figure 1: Photograph of the project taken in May, 2003. Four magnets seen in the foreground form the Small Isochronous Ring. Each magnet includes an aluminum Cshaped vacuum chamber with bi-metal flanges attached to the ends. C-shaped tubes of the two magnets closest to the injection point are connected to the vacuum chamber of the injection section. A piece of the injection line connects the injection section and the beam emittance measurement box seen in the background. The mass-state separator magnet with the ion source on top of it can be seen behind the box.

Table 1: Main SIR parameters

\begin{tabular}{lc}
\hline Beams & $H_{2}^{+}, D$ \\
Max. Energy & $30 \mathrm{keV}$ \\
$\beta(\mathrm{E}=20 \mathrm{keV})$ & $5 \cdot 10^{-3}$ \\
Bare tunes, $\nu_{x}, \nu_{y}$ & $1.15,1.11$ \\
Compaction factor, $\alpha_{p}$ & 1.0 \\
Circumference & $6.57 \mathrm{~m}$ \\
Lap time (E=20 keV) & $5 \mu \mathrm{s}$ \\
Number of turns & 30 \\
(limited by vacuum) & \\
Repetition rate & $100 \mathrm{~Hz}$ \\
& \\
Peak current & $20 \mu \mathrm{A}$ \\
Pulse Length (E=20 keV) & $50 \mathrm{~ns}-4 \mu \mathrm{s}$ \\
Emittance & $50 \pi \cdot \mathrm{mm} \cdot \mathrm{mrad}$ \\
Inj./Extr. energy spread & $0.1 \%-10 \%$ \\
SC tune shift & $0.05-0.2$ \\
\hline
\end{tabular}

by two fast semiconductor switches manufactured by Directed Energy, Inc. The switches are designed to drive capacitive loads and capable of generating $3.5 \mathrm{kV}$, rectangular pulses with a rise/decay time of $25 \mathrm{~ns}$.

The ion source, injection line, and injection section of the ring have been assembled and beam-tested. Paper [8] provides more details on a design and commissioning of the ions source, injection line, and injection system.
Dipole magnets, quadrupole correctors, and steering

Five identical dipole magnets are used for the project. Four magnets form the ring, and one is used as a mass-state separator in the injection line. Table 2 lists main parameters of the magnets.

Table 2: Magnet parameters

\begin{tabular}{lc}
\hline Gap & $71.4 \mathrm{~mm}$ \\
Bending angle & $90^{\circ}$ \\
Bending radius & $0.45 \mathrm{~m}$ \\
Magnetic field & 800 Gauss \\
Pole face angle & $26^{\circ}$ \\
Magnet weight & $210 \mathrm{~kg}$ \\
Power consumption & $700(x 5=3500) \mathrm{W}$ \\
\hline
\end{tabular}

All the dipole magnets have been assembled, installed on supports, and aligned. Because the magnets are all identical, we have mapped only one of the magnets in detail. To measure the field we used an NMR probe inside of the magnet and a Hall probe close to the edges. A normalized difference of the measured field from the field predicted by TOSCA did not exceed $3 \cdot 10^{-4}$ inside the magnet and $2 \%$ at the edges. The measured effective length of the magnet was larger than predicted by $2.54 \mathrm{~mm}$ or approximately $0.3 \%$. Using the measured field we calculated the betatron tunes in SIR. The radial tune $\nu_{x}$ had the same value in the measured field and in the field generated by TOSCA. The vertical tune $\nu_{y}$ calculated in the measured field was lower than $\nu_{y}$ calculated in the TOSCA field by 0.01 .

Each dipole magnet includes a gradient corrector coil and a dipole corrector coil. The final setup of the ring will also include four electrostatic quadrupoles and four vertical electrostatic deflectors.

\section{Vacuum system}

The vacuum chamber in all the dipoles is made of aluminum to avoid complications arising from variation of the $\mu$. The injection line and parts of vacuum chamber between magnets are made of 304 stainless steel. Aluminum and stainless steel parts are joined by bi-metal flanges manufactured by Atlas Technologies.

Two main sources of residual gas in the ring are outgasing from the walls of the vacuum chamber and the gas flux from the ion source. We plan to use three or four 500 $1 / \mathrm{s}$ turbo-pumps in the final setup with one or two pumps installed in the injection line and two pumps in the ring. Vacuum calculations show that pressure in the ring will be lower than $10^{-7}$ Torr if the ion source pressure is 1.0 mTorr. The expected beam life-time at this pressure, which is primarily determined by electron capture, is approximately 100 turns. 


\section{Diagnostics}

Diagnostics in the injection line consist of the beam emittance measurement box and a Faraday cup installed at the end of the line. Currently we use a "slow", unmatched version of the Faraday cup, which will be later substituted with a "fast" coaxial Faraday cup.

A retractable phosphor screen is situated in the injection section of the ring. It can be moved in one of the three positions: in the way of the beam coming from the injection line, in the median plane to detect the beam after a single turn, and below the median plane completely out of the way. The rest of diagnostics in SIR will include two vertical and two horizontal scanning wire monitors, two capacitive BPMs, and a movable fast Faraday cup. This Faraday cup will be used for measurement of the 2D longitudinalradial profiles of the beam with an accuracy of 1-2 mm.

\section{INJECTION LINE TEST}

The injection line and the injection section of the ring along with pieces of the vacuum chamber in two magnets closest to the injection point were assembled and pumped down to a vacuum of $5 \cdot 10^{-8}$ Torr after several days. The first attempt to run the beam through the injection line was successful. A $15 \mathrm{keV} \mathrm{H}_{2}^{+}$beam went through the injection line, was deflected into the median plane of the ring, and went through the first quarter of the ring. A Faraday cup situated after the first ring dipole registered a beam current of approximately $60 \mu \mathrm{A}$. The measured emittance of the beam was $15 \pi \cdot \mathrm{mm} \cdot \mathrm{mrad}$.

The beam chopper was tested in the regime of long bunches. It cut the DC beam into 5- $\mu$ sec-long pulses that were registered by the "slow", unmatched Faraday cup at the end of the injection line. A test of the capability of the chopper to generate short (50-ns-long) bunches requires a Fast Faraday cup with better time resolution.

\section{FUTURE PLANS}

Our future plans include:

- Construction

Design and assembly of remaining pieces of the vacuum chamber of the ring. Development, test, and installation of diagnostics in the ring, including a fast Faraday cup. Development of a control system and control software for the project.

- Commissioning

Injection in the ring, stable multi-turn operation, and measurement of beam parameters for low beam intensity, 1-5 $\mu \mathrm{A}$. Use of deuterium instead of hydrogen if the beam life-time is too short.

- Experiments, Phase I

Experiments on the longitudinal beam dynamics in the isochronous regime with tune shifts of 0.03-0.05.
Comparison of experimental results to multi-particle simulations.

- Experiments, Phase II

Experiments with high intensity beam in a nonisochronous regime. An expected tune shift at Phase II is 0.2 and larger. Operation of the ring with such a beam current may require installation of a simple RF system.

\section{ACKNOWLEDGMENT}

We would like to thanks J. Bierwagen for preparing drawings of SIR hardware. The authors would like also to express their gratitude to D. Pedtke for developing the ion source safety system. His expertise in electronics and helpful suggestions were crucial for a fast progress of the project. We are grateful to S. Hitchcock, D. Devereaux, and A. Mond for their assistance with assembling parts of the project.

\section{REFERENCES}

[1] Th. Stammbach et al., "The 0.9 MW Proton Beam at PSI and Studies on a 10 MW Cyclotron", Proc. 2nd Int. Conf. on Accelerator-Driven Transmutation Technologies, Kalmar 1996, p.1013

[2] Y. Alenitsky et al., "The High Current Cyclotron Complex for an Electro-nuclear Way of Production of Energy", Proc. 16th Int. Conf. on Cyclotrons and Their Applications, East Lansing 2001, p.434

[3] G.Kim, D. May, P. McIntyre, A. Sattarov, "A Superconducting Isochronous Cyclotron Stack as a Driver for a ThoriumCycle Power Reactor", Proc. 16th Int. Conf. on Cyclotrons and Their Applications, East Lansing 2001, p.437

[4] E. Pozdeyev, "Small Isochronous Ring for Experimental Study of the Longitudinal Space Charge Effect in Isochronous Cyclotrons", Proc. of PAC 2001 (Chicago), p. 3549-3551.

[5] E. Pozdeyev, F. Marti, J. Rodriguez, R. York, "Small Isochronous Ring Project at NSCL", Proc. of EPAC 2002 (Paris), p. 1395-1397.

[6] J. Rodriguez, E. Pozdeyev, F. Marti, "Injection Line of the Small Isochronous Ring”, Proc. of EPAC 2002 (Paris), p. 1401-1403.

[7] E. Pozdeyev, J. Rodriguez, "Computer Simulations of the Beam Dynamics in the Small Isochronous Ring”, Proc. of EPAC 2002 (Paris), p. 1398-1400.

[8] J. Rodriguez, F. Marti, E. Pozdeyev, "Initial Experimental Results of the Small Isochronous Ring”, these proceedings 